\title{
Metabolismo mineral en niños en diálisis peritoneal crónica
}

\author{
MARÍA LUISA CEBALLOS O. ${ }^{1}$, ANGÉLICA ROJO L. ${ }^{1}$, \\ MARTA AZÓCAR P. ${ }^{1}$, MARÍA JOSÉ IBACACHE M. ${ }^{1}$, ANGELA DELUCCHI B. ${ }^{1}$, \\ LILY QUIROZ Z. ${ }^{2}$, CARLOS IRARRÁZABAL M. ${ }^{3}$, IRIS DELGADO B. ${ }^{4}$, \\ FRANCISCA UGARTE P. ${ }^{3}$, FRANCISCO CANO SCH. ${ }^{1}$ \\ 1. Hospital Luis Calvo Mackenna. Facultad de Medicina, Universidad de Chile. \\ 2. Hospital Roberto del Río. \\ 3. Facultad de Medicina, Universidad de Los Andes. \\ 4. Facultad de Medicina Clínica Alemana, Universidad del Desarrollo.
}

\begin{abstract}
Mineral metabolism in patients on chronic peritoneal dialysis

Introduction: Children with chronic kidney disease (CKD) and receiving peritoneal dialysis (PD) have disorders of mineral metabolism that impact their growth, survival and cardiovascular functions. New molecular markers offer a better understanding of the pathophysiology of this disease. Objective: To characterize some components of mineral metabolism, with emphasis on FGF23/Klotho and cardiovascular functions (CV) of these patients. Patients and Method: Prospective observational cohort study. Exclusion criteria: serum 25 $(\mathrm{OH})$ vitamin $\mathrm{D}<20 \mathrm{ng} / \mathrm{ml}$, peritonitis within the last two months and active nephrotic syndrome. Calcemia, phosphemia, parathyroid hormone (PTH), $25(\mathrm{OH})$ vitD3, $1.25(\mathrm{OH})$ vitD3, FGF23 and Klotho in plasma were measured. FGF23 and Klotho were quantified in healthy children as a control group. Echocardiography was performed calculating the left ventricular mass index (LVMI). Descriptive statistics analysis, Pearson correlation coefficient for association among variables and multivariate analysis were conducted. Results: 33 patients, 16 males, aged between 1.2 and 13.4 years were included. Age of onset for PD: $7.3 \pm 5.0$ years, time receiving PD: $13.5 \pm 14.5$ months. The plasma concentration of $25(\mathrm{OH})$ vitD3 was $34.2 \pm 6.3 \mathrm{pg} / \mathrm{ml}$. Calcemia and phosphemia values were $9.8 \pm 0.71$ and $5.4 \pm 1.0 \mathrm{mg} / \mathrm{dl}$ respectively. PTH was $333 \pm 287 \mathrm{pg} / \mathrm{ml}$. FGF23 in plasma was $225.7 \pm 354.3 \mathrm{pg} / \mathrm{ml}$ and Klotho $131.6 \pm 72 \mathrm{pg} / \mathrm{ml}$, and in the controls $(\mathrm{n}=16$ ), it was $11.9 \pm 7.2$ $\mathrm{pg} / \mathrm{ml}$ and $320 \pm 119 \mathrm{pg} / \mathrm{ml}$, respectively. The residual and total dose of dialysis $(\mathrm{KtV})$ was $1.6 \pm 1.3$ and $2.9 \pm$ 1.6 , respectively. FGF23 levels significantly correlated with calcium $(\mathrm{p}<0.001, \mathrm{r}=0.85)$, and inversely with residual KtV, showing no relationship with phosphemia. Klotho level correlated negatively with residual KtV
\end{abstract}

Recibido el 17 de febrero de 2013, devuelto para corregir el 6 de septiembre de 2013, segunda versión 23 de septiembre de 2013, aceptado para publicación el 27 de noviembre de 2013.

Premio Mejor Trabajo Categoría General, 52 Congreso Chileno de Pediatría.

Financiamiento: Proyecto Fondecyt 1110226.

Este trabajo cumple con los requisitos sobre consentimiento /asentimiento informado, comité de ética, financiamiento, estudios animales y sobre la ausencia de conflictos de intereses según corresponda. 
and also, it showed a negative association with chronological age and age at onset of PD. LVMI $>38 \mathrm{~g} / \mathrm{m}^{2} \mathrm{was}$ confirmed in 20/28 patients. Conclusions: The values of FGF23, and PTH are elevated in children with CKD on PD. Klotho levels in CKD patients are lower than control children. A strong association of calcemia with FGF23 and PTH is reported. Residual renal function is inversely associated with FGF23 and Klotho. A high incidence of left ventricular hypertrophy was found evidencing a cardiovascular compromise in these patients. (Key words: Chronic kidney disease, mineral metabolism, bone disease, FGF23, Klotho).

Rev Chil Pediatr 2014; 85 (1): 31-39

\section{RESUMEN}

Introducción: Los niños portadores de Enfermedad renal crónica (ERC) en diálisis peritoneal (DP) presentan alteraciones del metabolismo mineral que afectan su crecimiento, estado cardiovascular y sobrevida. Nuevos marcadores moleculares representan una mejor comprensión de la fisiopatología de esta enfermedad. Objetivo: Caracterizar componentes del metabolismo mineral, con énfasis en FGF23/Klotho, y estado cardiovascular (CV) en este grupo de pacientes. Pacientes y Método: Estudio prospectivo observacional. Criterios de exclusión: niveles de $25(\mathrm{OH})$ vitamina $\mathrm{D}<20 \mathrm{ng} / \mathrm{ml}$, peritonitis hasta 2 meses previos y síndrome nefrótico activo. Se midió calcemia, fosfemia, paratohormona (PTH), $25(\mathrm{OH})$ vitD3, 1,25 (OH) vitD3, FGF23 y Klotho en plasma. Se cuantificó FGF23 y Klotho en niños sanos como grupo control. Se efectuó ecocardiografía, calculándose el índice de masa ventricular izquierda (IMVI). Se realizó análisis estadístico descriptivo, coeficiente de correlación de Pearson para asociación entre variables y análisis multivariado. Resultados: Se incluyeron 33 pacientes, 16 varones, edad 1,2 a 13,4 años. Edad de inicio de DP: 7,3 \pm 5,0 años, tiempo en DP: $13,5 \pm 14,5$ meses. El nivel plasmático de $25(\mathrm{OH})$ vitD3 fue $34,2 \pm 6,3 \mathrm{pg} / \mathrm{ml}$. Los valores de calcemia y fosfemia fueron 9,8 $\pm 0,71$ y $5,4 \pm 1,0 \mathrm{mg} / \mathrm{dl}$ respectivamente. La PTH fue de $333 \pm 287 \mathrm{pg} / \mathrm{ml}$. El FGF23 en plasma fue de $225,7 \pm 354,3 \mathrm{pg} / \mathrm{ml}$ y Klotho $131,6 \pm 72 \mathrm{pg} / \mathrm{ml}$, y en los controles $(\mathrm{n}=16)$ fue de $11,9 \pm 7,2 \mathrm{pg} /$ $\mathrm{ml}$ y $320 \pm 119 \mathrm{pg} / \mathrm{ml}$, respectivamente. La dosis de diálisis (KtV) residual y total fue de 1,6 $\pm 1,3$ y 2,9 \pm 1.6 , respectivamente. El nivel de FGF23 se correlacionó significativamente con la calcemia $(\mathrm{p}<0,001, \mathrm{r}=0,85)$, e inversamente con el KtV residual, sin mostrar relación con la fosfemia. El nivel de Klotho se correlacionó en forma negativa con el $\mathrm{KtV}$ residual y mostró una asociación negativa con la edad cronológica y la edad de inicio de DP. En 20/28 pacientes se confirmó un IMVI $>38 \mathrm{~g} / \mathrm{m}^{2}$. Conclusiones: Los valores de FGF23 y PTH se encuentran elevados en niños con ERC en DP. Los niveles de Klotho se encuentran disminuidos en estos pacientes en comparación a los controles. Destaca una fuerte asociación de la calcemia con FGF23 y PTH. La función renal residual se asoció inversamente a FGF23 y Klotho. Se constató una alta incidencia de hipertrofia ventricular izquierda evidenciando el compromiso cardiovascular en este grupo de pacientes.

(Palabras clave: Enfermedad renal crónica, metabolismo mineral, enfermedad ósea, FGF23, Klotho).

Rev Chil Pediatr 2014; 85 (1): 31-39

\section{Introducción}

El tratamiento de los niños con enfermedad renal crónica $(\mathrm{ERC})$ ha cambiado radicalmente en las últimas 2 décadas. El niño urémico con retraso de talla, anémico, hipertenso y con alteraciones osteoesqueléticas es una figura del pasado $^{1-3}$.

Los esfuerzos actuales se encaminan a comprender la fisiopatología de las complicaciones asociadas a la uremia, de forma de intervenir en ellas desde los inicios de la enfermedad. Nuevos marcadores moleculares como el Fac- tor de crecimiento fibroblástico 23 (en inglés, Fibroblast Growth Factor 23, FGF23) y su cofactor Klotho, han sido descritos en la última década, y su rol en los eventos fisiopatológicos de la uremia son objeto de investigación. En niños portadores de ERC el conocimiento sobre estos nuevos marcadores es muy limitado.

En 1997, Kuro-o y cols. ${ }^{4}$, y posteriormente White K. y cols. ${ }^{5}$ en pacientes portadores de Raquitismo Hipofosfatémico Autosómico Dominante (RHAD) identificaron al FGF23 como una hormona relacionada a la homeostasis del fósforo. Los altos niveles séricos de 
FGF23 en los pacientes portadores de RHAD condicionan un balance negativo de fosfato $\mathrm{y}$ la aparición de raquitismo ${ }^{6-8}$. En forma inversa, existen síndromes caracterizados por hiperfosfatemia y calcificaciones, asociados al déficit de FGF23. La Calcinosis Tumoral Familiar presenta este fenotipo, secundario a la mutación del gen GALNT3 que codifica una enzima requerida para la glicosilación del FGF23 y cuya deficiencia aumenta la susceptibilidad a la degradación del FGF23 sérico. En animales knock out FGF23 -/- se observa una severa retención de fosfato, hiperfosfemia y calcificación sistémica.

El FGF23 es una proteína de la familia de las fosfatoninas, de 250 aminoácidos, sintetizada fundamentalmente por los osteocitos. En el túbulo proximal, disminuyen la reabsorción de fosfato mediante la reducción de los niveles del cotransportador sodio-fosfato tipo 2a (Na$\mathrm{Pi}-2 \mathrm{a})$ en la membrana apical, generando fosfaturia, y por otra parte, suprime la expresión del gen Cyp27b1 que codifica para la enzima $1 \alpha$-hidroxilasa renal, responsable de la segunda hidroxilación y activación de la $25(\mathrm{OH})$ Vitamina D (vitD), reduciendo la síntesis de $1,25(\mathrm{OH})$ vitD. Igualmente aumenta la expresión del gen Cyp24 que codifica para la enzima 24-hidroxilasa, enzima que inactiva la vitamina D. Es así, un factor promotor de fosfaturiahipofosfemia, e inhibidor de la síntesis de 1,25 $(\mathrm{OH})$ vitD.

Recientes estudios han evaluado el rol del FGF23 y su relación con el metabolismo mineral óseo en enfermos renales. Wesseling-Perry y cols. ${ }^{9}$, han comunicado una experiencia en 52 pacientes portadores de ERC grados 2-4, con edades entre 2 y 21 años, evaluados con biopsia ósea. Los niveles de $25(\mathrm{OH})$ vitD y la fosfemia mostraron ser predictores de los valores de FGF23 en plasma, cifras elevadas en forma directamente proporcional a la caída de la función renal. Para los grados 2, 3, 4/5 de ERC se encontraron niveles de FGF23 de 181, 197 y 344 RU/ml (unidad de referencia/ $\mathrm{ml}$ ), respectivamente. Los niveles en plasma de FGF23 se encontraron directamente relacionados al score $\mathrm{Z}$ talla/edad, sugiriendo que esta hormona puede ser un factor asociado al retraso de talla en niños portadores de ERC.
En otro estudio de alto impacto clínico, Gutiérrez y cols. ${ }^{10}$, evaluaron la relación entre FGF23 y la mortalidad en 200 enfermos adultos en hemodiálisis y en 200 controles, encontrando un aumento significativo de FGF23 en los enfermos vs los controles (2.260 vs 1.406 $\mathrm{RU} / \mathrm{ml}$ ); el análisis multivariado mostró un aumento significativo de los valores de FGF23 a menor función renal, con una correlación positiva con la mortalidad de los enfermos. La relación de FGF23 y mortalidad fue independiente de los valores de fosfemia, variable sistemáticamente asociada a la mortalidad en pacientes portadores de ERC.

El FGF23 requiere de un cofactor para ejercer su efecto en el túbulo renal. El gen Klotho codifica una proteína de membrana similar a las enzimas beta-glucosidasas, fundamentales para el metabolismo celular, y por su rol en preservar la actividad celular ha sido asociada a la longevidad. Las ratas deficientes en FGF23 desarrollan hiperfosfemia y un fenotipo de senectud muy similar al de las ratas deficientes en Klotho, las cuales por su parte, al igual que en el déficit de FGF23, presentan hiperfosfatemia $^{11-13}$. Estas observaciones permitieron plantear la hipótesis de que ambas proteínas funcionaban en una vía común, con Klotho como co-receptor de FGF23 en la célula. Urakawa et al, en $2006^{14}$ y Nakatani y cols., en $2009^{15}$, observaron que ratas con niveles plasmáticos muy elevados de FGF23 pero deficientes en Klotho no presentaban los efectos del exceso de la fosfatonina, corroborando esta hipótesis.

Los estudios de metabolismo mineral que analizan las proteínas FGF23 y Klotho en pacientes pediátricos urémicos son muy escasos e inexistentes en nuestro país. El objetivo de este estudio es caracterizar algunos componentes del metabolismo mineral óseo en este grupo de pacientes, a través de los marcadores moleculares FGF23 y Klotho, estableciendo su relación con variables demográficas, nutricionales y bioquímicas.

\section{Pacientes y Método}

\section{Diseño}

Estudio prospectivo observacional, en pacientes portadores de insuficiencia renal en DP 
crónica de las Unidades de Nefrología Infantil de los hospitales Luis Calvo Mackenna y Roberto del Río. Los criterios de inclusión fueron: paciente estable desde punto de vista clínico y bioquímico; antecedente de haber sido recién nacido $>33$ semanas y adecuado para la edad gestacional, y adecuada adherencia familiar a la terapia. Como criterios de exclusión se consideró: trastornos de malabsorción gastrointestinal, enfermedades endocrinas, síndromes genéticos, síndrome nefrótico activo, peritonitis hasta 2 meses previos y niveles plasmáticos de $25(\mathrm{OH})$ vitD $<20 \mathrm{pg} / \mathrm{ml}$.

Se definió a un paciente como estable de acuerdo a la guías K-DOQI ${ }^{16-18}$ : Hemoglobina $11-12 \mathrm{~g} / \mathrm{dL}$, Bicarbonatemia $>22 \mathrm{mmol} / \mathrm{l}$, fosfemia 3,5-5,5 mg/dL, calcemia total 8,4-9,5 $\mathrm{mg} / \mathrm{dL}$, producto calcio-fósforo $<55$ y paratohormona (PTH) intacta entre $150-500 \mathrm{pg} / \mathrm{ml}$.

Todos los pacientes cumplieron el requisito de haber firmado el consentimiento o asentimiento informado. El protocolo fue aprobado por el Comité de Ética institucional de cada hospital, el Comité de Ética de la Facultad de Medicina de la Universidad de Chile, y el Comité de Ética de Fondecyt.

\section{Variables estudiadas}

Se evaluaron variables demográficas: edad, sexo, etiología de la ERC, edad de inicio de la DP y tiempo en DP. Todos los pacientes fueron tratados con DP cíclica automatizada mediante Baxter Home Choice PD System, con un volumen de intercambio de $1.100 \mathrm{ml} /$ $\mathrm{m}^{2}$ y concentraciones de dextrosa 1,5 a $4,25 \%$. Se registraron parámetros antropométricos en base a score Z: peso/edad, talla/edad. El peso fue determinado con balanza Seca (Seca Corporation, Hamburg, Germany) con $0,1 \mathrm{~kg}$ de precisión y $150 \mathrm{~kg}$ de peso máximo. La talla se midió con estadiómetro con $1 \mathrm{~mm}$ de precisión. La ingesta calórica y proteica $(\mathrm{g} / \mathrm{kg} / \mathrm{dí} a)$, de calcio y fósforo fue indicada de acuerdo a las guías K-DOQI $2008^{19}$, determinándose la adecuación a las recomendaciones (en porcentaje) y el nPNA (aparición de nitrógeno proteico normalizado). Se midieron los siguientes parámetros bioquímicos en sangre: creatininemia $(\mathrm{mg} / \mathrm{dl})$ por método enzimático, nitrógeno ureico $(\mathrm{mg} / \mathrm{dl})$, gases venosos, electrolitos plasmáticos, albúmina, calcemia, fosfemia, hemoglobina, hematocrito, ferritina, y PTH intacta (ensayo inmunoradiométrico, Nichols Institute, San Juan de Capistrano, CA, USA). Se cuantificó la $25(\mathrm{OH})$ vitD $_{3}$ y $1,25(\mathrm{OH})$ vitD $_{3}$ (RIA), y FGF23 y Klotho en plasma (Human Intact FGF-23 ELISA kit) en el Laboratorio de Biología Molecular del Hospital Luis Calvo Mackenna.

Se estableció como grupo control para FGF23 y Klotho a 16 niños con función renal normal, evaluados en el Laboratorio de Fisiología Integrativa del Instituto de Ciencias Biomédicas de la Universidad de Chile.

Se realizó ecocardiografía, calculándose el índice de masa ventricular izquierda (IMVI) en $\mathrm{g} / \mathrm{m}^{2}$ (fórmula $=1,04 \times[(\mathrm{SIV}+\mathrm{DFDVI}+$ $\mathrm{PP})^{3}-(\mathrm{DFDVI})^{3}+0,6$, por factor corrección $0,8)$, con un valor normal $<38 \mathrm{~g} / \mathrm{m}^{2}$.

\section{Análisis estadístico}

Los datos fueron evaluados mediante el programa Shapiro-Wilks statistic, las variables de distribución normal se expresaron en promedios y desviaciones estándar. Aquellas con distribución no normal, en medianas y rangos. Las diferencias entre grupos de distribución normal fueron evaluadas por $t$ tests, y las no normales con Wilcoxon sign-rank tests. Se utilizó coeficiente de correlación de Pearson para determinar asociación entre variables y análisis multivariado para establecer correlación entre variables. Un $p<0,05$, se consideró significativo.

\section{Resultados}

Se evaluaron 33 pacientes (16 varones), con edades entre 1,2 y 13,4 años (promedio 8,3 años). La edad de inicio de DP fue 7,3 $\pm 5,0$ años y el tiempo en DP fue de 13,5 $\pm 14,5$ meses, mediana 10 meses. Las etiologías de ERC fueron: displasia renal en 8 pacientes, uropatía obstructiva en 7 , síndrome nefrótico en 6 , nefropatías hereditarias en 4 , desconocido en 3 , síndrome hemolítico urémico en 3 y vasculitis en 1 paciente. Se observó un promedio score $Z$ peso/edad de $-1,18 \pm 1,22$ y $Z$ talla/edad $-2,07$ $\pm 1,16$. Al evaluar la ingesta calórica y proteica 
se obtuvo una adecuación de un 92,3\% (rango $69-126 \%$ ) y $116,1 \%$ (rango $72,5-190 \%$ ), respectivamente. La adecuación de la ingesta para Calcio fue de $81,3 \%$ (rango $31,1-174 \%$ ) y para fósforo fue 99,6\% (rango 53,3-326\%).

Los valores promedio de calcemia y fosfemia fueron $9,8 \pm 0,71$ y $5,4 \pm 1,0 \mathrm{mg} / \mathrm{dl}$, respectivamente. La PTH intacta fue de 333 $\pm 287 \mathrm{pg} / \mathrm{ml}$. El nivel plasmático de $25(\mathrm{OH})$ vitD fue $34,2 \pm 6,3 \mathrm{pg} / \mathrm{ml}$. La 1,25 $(\mathrm{OH})$ vitD $_{3}$ fue de $23 \pm 20 \mathrm{pg} / \mathrm{ml}$. El valor promedio de FGF23 y Klotho fue de 225,7 $\pm 354,3$ y 131,6 $\pm 72 \mathrm{pg} / \mathrm{ml}$, respectivamente (tabla 1). En el grupo control (edad 6,3 $\pm 2,1$ años), los valores de FGF23 y Klotho fueron $11,95 \pm 7,2 \mathrm{pg} / \mathrm{ml}$ y $320 \pm 119 \mathrm{pg} / \mathrm{ml}$, respectivamente.

Al evaluar el nivel de FGF23 por rango de edad, se observó en los segmentos $<2,2-12$ y $>12$ años, valores de $253 \pm 195,202 \pm 377$ y $257 \pm 376 \mathrm{pg} / \mathrm{ml}$, respectivamente, sin diferen- cias entre los grupos.

El promedio de dosis de diálisis $(\mathrm{KtV})$ residual y total fue de $1,6 \pm 1,3$ y $2,9 \pm 1,6$, respectivamente.

Los niveles de PTH intacta y la calcemia, en el análisis multivariado presentaron una asociación negativa significativa $(\mathrm{p}=0,01$, $r=-0,45$ ), en tanto que la fosfemia y PTH mostraron una asociación lineal positiva significativa $(\mathrm{p}=0,02, \mathrm{r}=0,41)$ (figuras $1 \mathrm{a}$ y $1 \mathrm{~b})$.

El nivel plasmático de FGF23 se correlacionó en forma significativamente positiva con la calcemia ( $\mathrm{p}<0,001, \mathrm{r}=0,82)$ (figura 2a), pero no con la fosfemia (figura 2b). El nivel de Klotho se asoció en forma negativa con el KtV residual, con la edad cronológica y de inicio de DP.

En 20/28 pacientes se confirmó un IMVI $>38 \mathrm{~g} / \mathrm{m}^{2}$, sin presentar correlación con FGF23 ni Klotho.

Tabla 1. Marcadores del metabolismo mineral grupo estudio según rango etario, en pacientes pediátricos con diálisis peritoneal crónica

\begin{tabular}{|ccccccc|}
\hline $\begin{array}{c}\text { Rango edad } \\
\text { (años) }\end{array}$ & $\begin{array}{c}\text { n pacientes } \\
\text { (hombres) }\end{array}$ & $\begin{array}{c}\text { Calcemia } \\
\text { (mg/dl) }\end{array}$ & $\begin{array}{c}\text { Fosfemia } \\
\text { (mg/dl) }\end{array}$ & $\begin{array}{c}\text { PTH } \\
\text { (pg/ml) }\end{array}$ & $\begin{array}{c}\text { FGF23 } \\
\text { (pg/ml) }\end{array}$ & $\begin{array}{c}\text { Klotho } \\
\text { (pg/ml) }\end{array}$ \\
\hline 2 & $4(3)$ & $9,25 \pm 1,28$ & $5,2 \pm 1,52$ & $466 \pm 432$ & $253 \pm 195$ & $132 \pm 0,48$ \\
\hline $2-12$ & $15(7)$ & $9,97 \pm 1,05$ & $5,5 \pm 0,99$ & $324 \pm 275$ & $202 \pm 377$ & $121 \pm 18,6$ \\
\hline 12 & $14(6)$ & $10,05 \pm 1,29$ & $5,71 \pm 1,04$ & $336 \pm 285$ & $257 \pm 376$ & $139 \pm 103$ \\
\hline
\end{tabular}

PTH: Paratohormona.
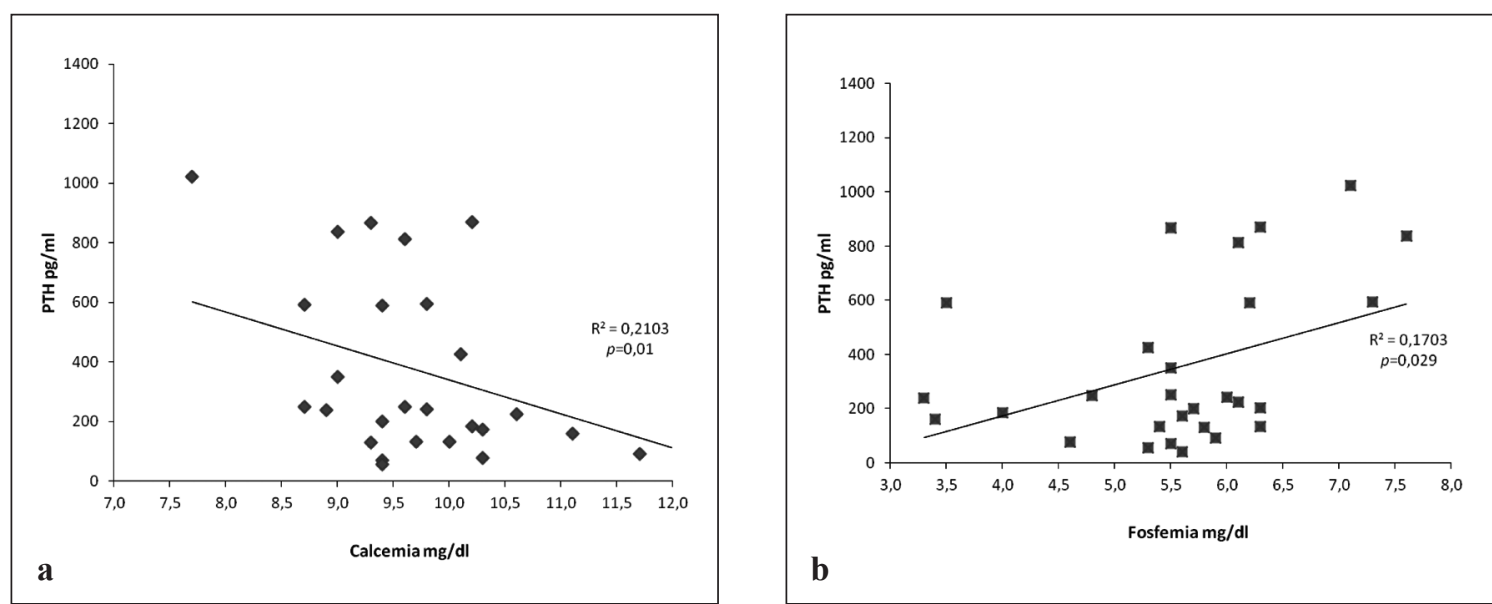

Figura 1. Correlación entre paratohormona y calcemia (a) y paratohormona vs fosfemia (b) en pacientes pediátricos con diálisis peritoneal crónica. 

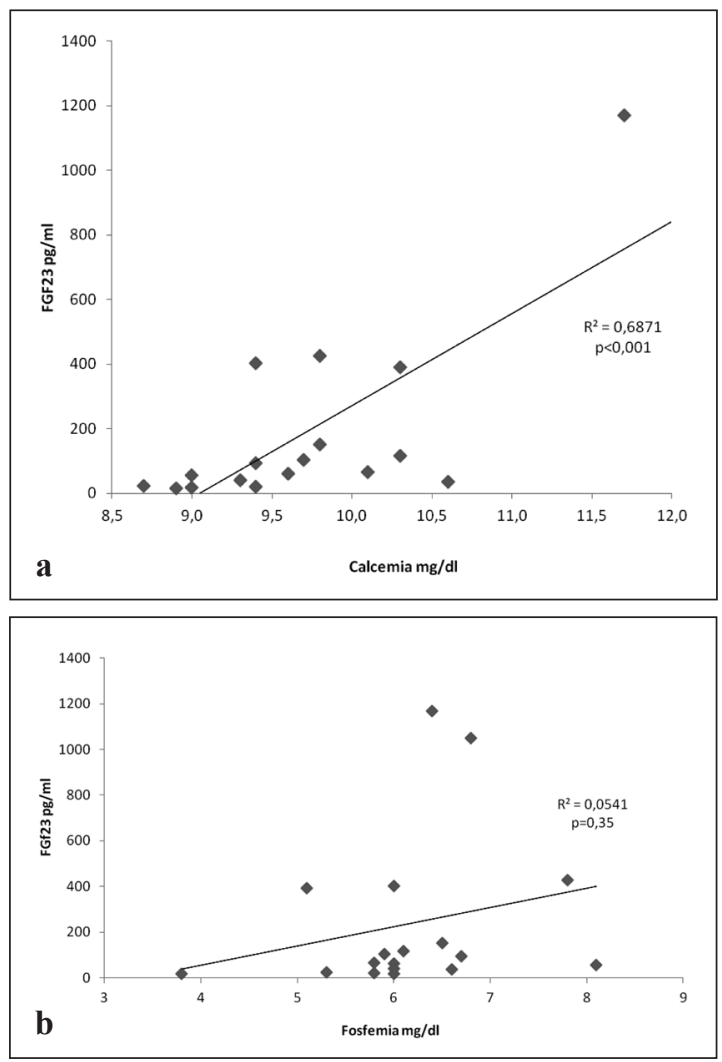

Figura 2. Correlación entre FGF23 y calcemia (2a) y FGF23 $v s$ fosfemia (2b), en pacientes pediátricos con diálisis peritoneal crónica.

\section{Discusión}

La regulación del metabolismo mineral óseo en la ERC ha sido exhaustivamente estudiada dado su impacto en la morbimortalidad de estos pacientes. El descubrimiento de las proteínas FGF23 y su cofactor Klotho, representa un importante avance en esta patología. E1 FGF23 produce fosfaturia y frena la síntesis de 1,25 (OH) vitD en el riñón, en un efecto que se ha planteado corrige el potencial exceso de fosfato producto de la acción de la 1,25 $(\mathrm{OH})$ vitD $_{3}$ que se genera frente a la hipocalcemia, propia de la ERC. La hipocalcemia gatilla una respuesta en la PTH, la cual moviliza calcio y fósforo del hueso, al mismo tiempo que estimula la producción renal de $1,25(\mathrm{OH})$ vitD $_{3}$, hormona que estimula la reabsorción de calcio $y$ fosfato en el intestino. El exceso de fosfato producto de este eje activado, requiere de un factor corrector, que el FGF23 explica en forma satisfactoria. La producción de FGF23 en los osteoblastos y osteocitos se ha relacionado a una alta ingesta de fosfatos, a pesar de que la elevación del fosfato plasmático no ha sido claramente demostrado, aumente los niveles de FGF23 en individuos sanos ${ }^{21}$. En un estudio, se analizó la adecuación de fósforo a partir de la medición de la ingesta y de las recomendaciones, encontrando un valor porcentual de 99,6 \pm 56,8. La fosfemia promedio se encontró dentro de las recomendaciones KDO$\mathrm{QI}^{18}$ para niños de hasta 12 años. Antoniucci y cols. ${ }^{22}$, demostraron en un estudio controlado en 13 adultos sanos, una relación directa entre la ingesta de fosforo de la dieta y los niveles de FGF23, relación que en este estudio no fue observada. Sí pudimos confirmar una correlación positiva entre adecuación de fósforo $v s$ ingesta proteica y nPNA, reforzando la importancia de la dieta y control de ingesta proteica para el manejo del fósforo.

La adecuación de ingesta de calcio mostró un valor de $81,3 \pm 39 \%$, con una calcemia promedio que supera el límite de la recomendación KDOQI 2005. La PTH evidenció una cifra promedio dentro de las recomendaciones pediátricas. Esta hormona presentó una correlación positiva significativa con el nivel de fósforo sérico y negativa con la calcemia, en una interacción característica de la ERC etapa 5. En efecto, el análisis multivariado reveló que el cambio de una unidad en la calcemia produce una disminución de PTH de 113 unidades (IC 95\% -194;-32), y una unidad de cambio en la fosfemia explicó un aumento de 114 unidades en la PTH (IC 95\% 24; 204). No se demostró sin embargo, ninguna correlación significativa entre la PTH y FGF23, lo cual resulta comprensible dado el efecto supresor sobre la PTH que se ha descrito para la FGF23. Wesseling-Perry y cols. ${ }^{9}$, han sugerido una relación significativa entre PTH y FGF23 dependiente del nivel de filtración glomerular, sin embargo, el bajo número de pacientes incluidos en ese estudio en el grupo ERC etapa 4/5 (n: 14) requiere una confirmación en un mayor número de pacientes. Imanishi y cols. ${ }^{23}$, demostraron la correlación directa entre FGF23 y PTH en pacientes en hemodiálisis, sin embargo, los pa- 
cientes eran adultos mayores y sus resultados deben ser extrapolados con cautela a la población pediátrica.

Los valores de FGF23 en la población estudiada revelaron una mediana significativamente mayor al valor promedio observado en el grupo control, con un valor cercano al publicado por Shimada T y cols. ${ }^{8}$, de $20,03 \pm$ $5,14 \mathrm{pg} / \mathrm{ml}$. Isakova y cols. ${ }^{24}$, caracterizaron los niveles de FGF23 en adultos con distinto grado de filtración glomerular. En 87 pacientes etapa 5 comunicaron una mediana de $348 \mathrm{pg} /$ $\mathrm{ml}$ (rango 234-486), en tanto que WesselingPerry y cols., publicaron una mediana de 344 $\mathrm{RU} / \mathrm{ml}$ (255-742) en 14 niños con ERC etapas $4 / 5$ de insuficiencia renal. En este estudio, 2 pacientes evidenciaron niveles de FGF23 sobre $1.000 \mathrm{pg} / \mathrm{ml}$, cifras que explican la amplia distribución de la muestra, y sin los cuales la mediana obtenida es de 63,3 , con un rango de 17 a 426 pg/ml. Actualmente deben precisarse las unidades de los valores usados para medir la FGF23, dado que existen 2 kits disponibles. El primero de ellos usa 2 anticuerpos monoclonales que reconocen la porción $\mathrm{N}$ y C-terminal de la proteína de 251 aminoácidos, regiones 25-179 y 180-251 respectivamente, conocido como método iFGF23 (intactFGF23, Kainos, Tokio, Japan) y expresado en unidades pg/ $\mathrm{ml}$. El segundo método usa 2 anticuerpos que reconocen epítopes localizados en la región C-terminal de la proteína, residuos 206-222 y 225-244, conocido como método C-Terminal (Immunotopics, San Clemente, CA, USA) y expresado en unidades RU/ml. En un estudio que comparó ambos métodos en 34 pacientes en DP, 13 niños, Shimada y cols..$^{25}$, demostraron una correlación lineal positiva entre ambas mediciones, con un $r=0,92$, al mismo tiempo confirmaron que prácticamente el $100 \%$ de la hormona circulante en plasma existe en la forma intacta y biológicamente activa.

La relación entre FGF23 y fosfemia ha sido comunicada en diferentes estudios. En niños, Wesseling-Perry y cols ${ }^{9}$ demostraron que la $25(\mathrm{OH})$ vitD y la fosfemia son predictores independientes de los niveles de FGF23, sin embargo, ese estudio incluyó pacientes con ERC etapas 2-5, y los 14 pacientes en etapa $4 / 5$ tuvieron una fosfemia sensiblemente ma- yor que nuestra población. El rol del fósforo en la génesis de la elevación del FGF23 es controversial. Isakova T y cols. ${ }^{24}$ evaluaron 3.879 pacientes del Chronic Renal Insufficiency Cohort Study, que incluye pacientes portadores de ERC 2-4; los valores promedio de fosfemia y PTH se encontraban normales, pero los niveles de FGF23 al tiempo equivalente se encontraban francamente elevados. Esta observación se suma al hallazgo de valores disminuidos de fósforo plasmático y altos niveles de FGF23, en pacientes portadores de Enfermedad Renal Poliquística Autosómica Dominante. Los autores concluyen que esta proteína podría funcionar como un precoz marcador de la caída de la filtración glomerular, y comunican una asociación significativa positiva entre los niveles de FGF23 y la PTH y el fosfato sérico en un subgrupo de 332 pacientes, sin especificar su nivel de filtración glomerular. Estos autores destacan que no todos los pacientes portadores de ERC presentan valores elevados de FGF23, hallazgo que es compartido por nuestro estudio. Además, proponen que factores genéticos, la severidad del compromiso renal, y una alta ingesta de fosfatos, asociada a una baja condición socioeconómica, pudiesen explicar la variabilidad de los resultados, fenómeno que se repite en diferentes series analizadas. En nuestra experiencia, no encontramos asociación entre la fosfemia y los niveles de FGF23, lo cual podría explicarse por la homogeneidad de esta población pediátrica en términos de ingesta adecuada de fósforo, un nivel de fosfemia dentro de las recomendaciones y con escasa variabilidad, producto de la fuerte manipulación terapéutica dietética y farmacológica dirigidas al control del fósforo en diálisis pediátrica. En cambio, en forma inesperada, se demostró una fuerte asociación entre calcemia y FGF23. El análisis multivariado de estas relaciones evidenció que por cada unidad de cambio de la calcemia hay un cambio de 285 unidades del FGF23 (IC 95\%, 183-386). El análisis del cofactor Klotho mostró cifras inferiores al grupo control, sin encontrarse asociación con FGF23 ni las otras variables bioquímicas estudiadas. Sugiura H. y cols. ${ }^{26}$, midieron la expresión del Klotho soluble mediante un kit ELISA en pacientes portadores de ERC y en controles, con- 
firmando que esta proteína se encuentra disminuida en los pacientes urémicos, sin demostrar correlación entre los resultados de FGF23 y este cofactor. En nuestro estudio este cofactor mostró una asociación significativa inversa con la edad de los pacientes y con el KtV residual, una asociación que requiere ser analizada en forma particular.

En la evaluación ecocardiográfica, se observó una alta incidencia de hipertrofia ventricular izquierda (LMVI). Seeherunvong y cols $^{27}$ publicaron el año 2012, un estudio retrospectivo en 26 jóvenes (6-21 años) portadores de ERCT a quienes se efectuó ecocardiografía, encontrado un 55\% de HIV con una correlación positiva con FGF23. Aunque en nuestro grupo no se evidenció este efecto, la alta incidencia de HVI, revela la importancia del compromiso cardiovascular subclínico en este grupo de niños.

Los hallazgos de este estudio revelan que la importante elevación de FGF23 son propios de la ERCT (etapa 5), iniciándose sin embargo, su ascenso en etapas anteriores de la enfermedad renal crónica, como se comentó anteriormente. Esta elevación, responde inicialmente a la necesidad de aumentar la excreción de fósforo $\mathrm{y}$ al mismo tiempo, frenar su absorción, dado que con la progresiva caída de la velocidad de filtración glomerular, la acumulación de fósforo requiere de mecanismos compensatorios a ambos niveles. En este sentido el FGF23, ejerce una eficaz acción sobre la excreción de fósforo, aunque limitada por la función renal residual, lo cual puede explicar el significativo aumento de los niveles plasmáticos de esta hormona en los pacientes en diálisis, caracterizados por una progresiva pérdida de la función renal residual. En este mismo sentido, el FGF23 complementa su acción reguladora de la fosfemia a través de la supresión de la 1a hidroxilasa, limitando la absorción intestinal de calcio y fósforo, y por otro lado disminuyendo el recambio óseo, mediante la inhibición de la PTH. Estas últimas dos acciones del FGF23, que hipotéticamente podrían representar un intento por mantener su efecto a pesar de una progresiva caída de filtración glomerular, tienen como consecuencia la generación de una hipocalcemia, por lo que no resulta extraño que los niveles plasmáticos de calcio sean resguardados a través de un feedback directo entre la calcemia y el FGF23, tal como se desprende de los hallazgos presentados en este estudio.

En conclusión, en niños en DP crónica, la proteína FGF23 se encuentra aumentada y klotho disminuida, con respecto a los valores de niños normales, complementando la información clásica aportada por la paratohormona, calcemia y fosfemia. Destaca en esta experiencia la importancia de la dieta en el control de la fosfemia, la interrelación entre PTH, calcio $\mathrm{y}$ fósforo, $\mathrm{y}$ en forma preliminar comunicamos una importante asociación entre FGF23 y calcemia, correlación que requiere ser evaluada en un estudio prospectivo con un mayor número de sujetos.

\section{Referencias}

1.- North American Pediatric Renal Transplant Cooperative Study (2006) Annual report. Disponible en: http:// web.emmes.com/study/ped/annlrept/annlrept2006.pdf

2.- Moe S, Dru T, Cunningham G, et al: Definition, evaluation, and classification of renal osteodystrophy: A position statement from Kidney Disease: Improving Global Outcomes (KDIGO) disponible en: http://www. kdigo.org/meetings_events/pdf/moe_etal_KI_2006.pdf

3.- Slatopolsky E, Brown A, Dusso A: Role of phosphorus in the pathogenesis of secondary hyperparathyroidism. Am J Kidney Dis 2001; 37 (1 Suppl 2): S54-S57.

4.- Kuro-o M, Matsumura Y, Aizawa H, et al: Mutation of the mouse klotho gene leads to a syndrome resembling ageing. Nature 1997; 6; 390 (6655): 45-51.

5.- White KE, Evans WE, O'Riordan JLH, et al: Autosomal dominant hypophosphataemic rickets is associated with mutations in FGF23. Nat Genet 2000; 26: 345-8.

6.- Rowe P: The wrickkened Pathways of FGF23, MEPE and PHEX. Crit Rev Oral Biol Med 2004; 15: 264-81.

7.- Quarles L: FGF23, PHEX, and MEPE regulation of phosphate homeostasis and skeletal mineralization. Am J Physiol Endocrinol Metab 2003; 285: 1-9.

8.- Shimada T, Mizutani S, Muto T, et al: Cloning and characterization of FGF23 as a causative factor of tumor induced osteomalacia. Proc Natl Acad Sci 2001; 98: 6500-5

9.- Wesseling-Perry K, Pereira $R$, Wang $H$, et al: Relationship between Plasma Fibroblast Growth Factor 23 concentration and Bone Mineralization in Children with Renal Failure in Peritoneal Dialysis. J Clin Endocrinol 
Metab 2009; 94: 511-7.

10.- Gutiérrez O, Mannstadt M, Isakova T, et al: Fibroblast Growth Factor 23 and Mortality among Patients Undergoing Hemodialysis. N Engl J Med 2008; 359: 584-92.

11.- Imura Y, Tsuji Y, Murata M, et al: Alpha-Klotho as a regulator of calcium homeostasis. Science 2007; 316: 1615-8.

12.- Kuro-o M, Matsumura Y, Aizawa H, et al: Mutation of the mouse klotho gene leads to a syndrome resembling ageing. Nature 1997; 390: 45-51.

13.- Tsujikawa H, Kurotaki Y, Fujimori T, Fukuda K, Nabeshima $Y$ : Klotho, a gene related to a syndrome resembling human premature aging, functions in a negative regulatory circuit of vitamin D endocrine system. Mol Endocrinol 2003; 17: 2393-403.

14.- Urakawa I, Yamazaki Y, Shimada T, et al: Klotho converts canonical FGF receptor into a specific receptor for FGF23. Nature 2006 7; 444: 770-4.

15.- Nakatani T, Ohnishi M, Razzaque MS: Inactivation of klotho function induces hyperphosphatemia even in presence of high serum fibroblast growth factor 23 levels in a genetically engineered hypophosphatemic (Hyp) mouse model. FASEB J 2009; 23: 3702-11.

16.- Waller S, Ledermann S, Trompeter $R$, van't Hoff $W$, Ridout D, Rees $L$ : Catch-up growth with normal parathyroid hormone levels in chronic renal failure. Pediatr Nephrol 2003; 18: 1236-41.

17.- $N K F-K / D O Q I$ Clinical Practice Guidelines for Anemia of Chronic Kidney Disease: update 2000. Am J Kidney Dis 2001; 37 (1 Suppl 1): S182-S238.

18.- KDOQI Clinical Practice Guidelines for Bone Metabolism and Disease in Chronic Kidney Disease. Am J Kidney Dis 2003; 42, (4 Suppl 3): S1-S201.
19.- KDOQI Clinical Practice Guideline for Nutrition in Children with CKD: 2008 Update. Am J Kidney Dis 2009; 53, (3 Suppl 2): S1-S124.

20.- Cano F, Azócar M, Marín V, et al: Dosis de diálisis, nutrición y crecimiento en diálisis peritoneal pediátrica. Rev Med Chile 2005; 133: 1455-64.

21.- Ferrari SL, Bonjour JP, Rizzoli R: Fibroblast growth factor-23 relationship to dietary phosphate and renal phosphate handling in healthy young men. J Clin Endocrinol Metab 2005; 90: 1519-24.

22.- Antoniucci DM, Yamashita T, Portale AA: Dietary phosphorus regulates serum fibroblast growth factor-23 concentrations in healthy men. J Clin Endocrinol Metab 2006; 91: 3144-9.

23.- Imanishi $Y$, Inaba $M$, Nakatsuka $K$, et al: FGF-23 in patients with end-stage renal disease on hemodialysis. Kidney Int 2004; 65: 1943-6.

24.- Isakova T, Wahl P, Vargas G, et al: Fibroblast growth factor 23 is elevated before parathyroid hormone and phosphate in chronic kidney disease. Kidney Int 2011, $79 ; 1370-8$.

25.- Shimada T, Urakawa I, Isakova I, et al: Circulating Fibroblast Growth Factor 23 in Patients with End-Stage Renal Disease Treated by Peritoneal Dialysis Is Intact and Biologically Active. J Clin Endocrinol Metab 2010; 95: 578-85.

26.- Sugiura H, Tsuchiya K, Nitta K: Circulating levels of soluble a-Klotho in patients with chronic kidney disease. Clin Exp Nephrol 2011; 15: 795-6.

27.- Seeherunvong $W$, Abitbol C, Chandar Y, Rusconi P, Zilleruelo, Freundlich M: Fibroblast growth factor 23 and left ventricular hypertrophy in children on dialysis. Pediatr Nephrol 2012; 27: 2129-36. 